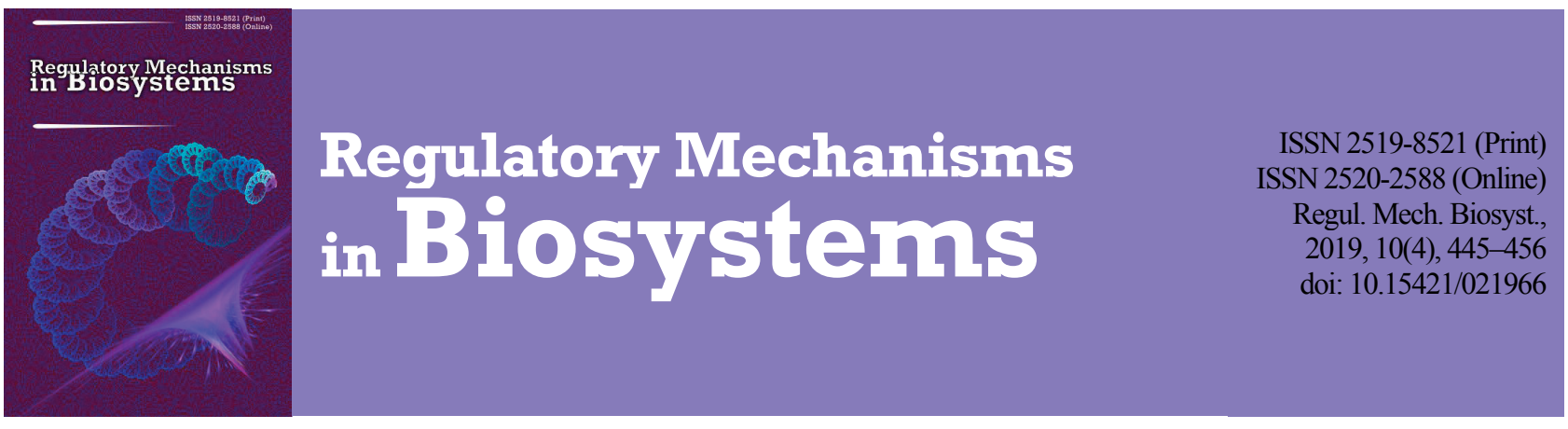

\title{
Synergistic activity of filtrates of Lactobacillus rhamnosus and Saccharomyces boulardii and antibacterial preparations against Corynebacterium spp.
}

\author{
O. Y. Isayenko \\ I. I. Mechnikov Institute of Microbiology and Immunology of National Academy of Medical Sciences of Ukraine, Kharkiv, Ukraine
}

Article info

Received 16.09.2019

Received in revised form 20.10.2019

Accepted 21.11.2019

I. I. Mechnikov Institute of Microbiology and Immunology of National Academ of Medical

Sciences of Ukraine,

Pushkinska st., 14/16, Khar-

kiv, 61057, Ukraine.

Tel.: +38-099-434-18-30

E-mail:el_isaenko@ukr.net

\section{Introduction}

Antimicrobial biologically active substances, besides being safe, are highly active, making them promising therapeutic agents for the treatment of diseases of different genesis and the creation of candidate drugs for combination therapy (Pfalzgraff et al., 2018). Combined use of synthetic peptides with the antibiotics ciprofloxacin, meropenem, erythromycin, gentamicin and vancomycin is known to improve healing of skin abscesses caused by Enterococcus faecium, Staphylococcus aureus, Klebsiella pneumoniae, Acinetobacter baumannii, Pseudomonas aeruginosa, Enterobacter cloacae and Escherichia coli. Their combined treatment is accompanied by a significant reduction in the size of the abscess irrespective of the mode of action of the antibiotic (Pletzer et al., 2018). Increase in the effectiveness of existing antibacterial drugs, the range of action of which is limited by the permeability barrier represented by the outer membrane of gram-negative microorganisms (efflux pump), was achieved by Corbett et al. (2011). The combination of SPR741 with antibiotics was tested for Escherichia coli, Klebsiella pneumoniae and Acinetobacter baumannii. Potentiation was achieved with clarithromycin, erythromycin, fusidic acid, retapamulin and rifampin against $A$. baumannii. The highest
Isayenko, $O$. Y. (2019). Synergistic activity of filtrates of Lactobacillus rhamnosus and Saccharomyces boulardii and antibacteri-
al preparations against Corynebacterium spp. Regulatory Mechanisms in Biosystems, 10(4), 445-456. doi:10.15421/021966

We present the results of the first study of the combined influence of the biologically active substances Lactobacillus rhamnosus GG ATCC 53103 and Saccharomyces boulardii, obtained by the author's method, and antibacterial agents on Corynebacterium spp. consecutive effects of the structural components and metabolites of $L$. rhamnosus GG and $S$. boulardii and antibacterial drugs on Corynebacterium spp. tox ${ }^{+}$. The greatest increase in the sensitivity of test-cultures of corynebacteria to penicillin (by $19.4 \mathrm{~mm}$ ), imipenem (by $15.0 \mathrm{~mm}$ ), vancomycin (by $12.0 \mathrm{~mm}$ ), gentamicin (by $11.0 \mathrm{~mm}$ ), ciprofloxacin (by $9.8 \mathrm{~mm}$ ), erythromycin (by $9.6 \mathrm{~mm}$ ), romycetes. The second area of research was the study of the synergic activity of substances L. rhamnosus GG and S. boulardii and traditional antibacterial drugs manifested by their simultaneous effect on Corynebacterium spp. Maximum potentiation of azithromycin (by $4.6 \mathrm{~mm}$ ), erythromycin (by $4.5 \mathrm{~mm}$ ), cefotaxime (by $2.2 \mathrm{~mm}$ ), ceftriaxone (by $1.6 \mathrm{~mm}$ ) and ampicillin (by $1.0 \mathrm{~mm}$ ) relative

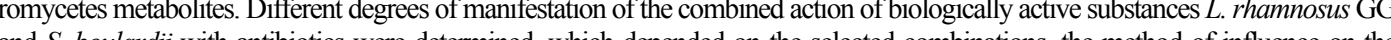
ers used to obtain the products of vital activity of lactobacteria and saccharomyces. The presented complexes of structural componetabolites of L. rhamnosus GG and S. boulardii, obtained without the use of traditional nutrient media, by increasing the "accompaniment-preparations" for antibiotics for the additional therapy of infectious diseases of different etiology, and for the creation of

Keywords: biologically active substances; disintegrates and metabolites of lactobacteria; saccharomycetes with antibiotics; sensitivity of Corynebacterium spp;; potentiation of antibacterial preparations.

efficacy of the antibiotic-SPR741 combinations was found for up to 25 polyresistant and clinical E. coli and K. pneumoniae strains and 17 A. baumannii test cultures. Research has shown the possibility of further combined use of SPR741 with antibacterial agents for the treatment of bacterial infections caused by gram-negative microorganisms (Corbett et al., 2017). The antimicrobial activity of the synthetic N-terminal lactoferrin peptide (hLF1-11) in combination with antibiotics (gentamicin, tigecycline, rifampicin, clindamycin, clarithromycin) was evaluated with respect to polyresistant strains of Klebsiella pneumoniae with different carbapenemase genes (OXA-48, KPC-2, KПК-3, BIM-1). The synergistic effect of hLF1-11 with antibiotics tested has been established and the effectiveness of their combined use for the treatment of infections caused by Klebsiella pneumoniae with multiple drug resistance has been demonstrated (Morici et al., 2018).

Studies by many authors have proved the effectiveness of the use of antimicrobial substances LL-37, bufferin II, ceprocine P1, machinin II with antibacterial drugs - polymyxin, piperacillin, azithromycin, daptomycin, linezolid, clarithromycin. Combined use is accompanied by an increase in the therapeutic effect of antibiotics against gram-negative, grampositive microorganisms, as well as against pathogens with high multiple 
drug resistance (Pizzolato-Cezar et al., 2019). The potentiation of rifampin, meropenem, aztreonam, tobramycin was due to SET-M33 against the multiresistant strains of Klebsiella pneumoniae, Acinetobacter baumannii, and ciprofloxacin was also noted against $P$. aeruginosa (Pollini et al., 2017). Synergistic action of azithromycin and vancomycin was established in conjunction with DP7 against resistant pathogens Staphylococcus aureus, P. aeruginosa and Escherichia coli (Wu et al., 2017).

Despite the large number of works on enhancing the antimicrobial activity of antibacterial drugs with biologically active peptide substances of synthetic origin, there are also opposite data. Results of independent studies have found insufficient synergy between synthesized antimicrobial agents and antibacterial drugs. The authors synthesized, purified (Biosynthesis, Inc.) and identified using high performance liquid chromatography three known peptides (VAYR $=$ RRGWVLALYLRYGRR, ARVA = RRGWLALRLVLAY, VVRG = WVLVLRLGY). The fourth object taken for the study was the highly active protein melittin (the main active substance of bee venom). Despite its high toxicity to human cells and bacteria, the assessment of synergistic interaction with antibiotics has been made due to its ability to penetrate the membranes of various microorganisms (bacteria and fungi). Antimicrobial peptides taken for the experiment previously have been characterized by the violation of the membranes of gram-negative and gram-positive bacteria in combination with antibiotics. The studies were performed on four antimicrobial agents in combination with four different peptides against three types of gram-negative and gram-positive bacteria (40 total pairwise measurements) and were expressed as fractional inhibitory concentration. The authors conclude that none of the antimicrobial substances characterized by penetration into bacterial membranes enhanced the antimicrobial action of antibiotics (ampicillin, ciprofloxacin, streptomycin vancomycin) for all test-strains (E. coli ATCC 25922, P. aeruginosa ATCC 27853, S. aureus ATCC 25923). Therefore, destruction of membranes and penetration of peptides of synthetic origin into microbial cells is not sufficient for synergistic interaction with low molecular weight antibiotics against gram-negative or gram-positive microorganisms. At combination treatment with lipopeptide Bacillomycin $\mathrm{D}$ and antifungal amphotericin $\mathrm{B}$ antimicrobial and wound healing effect was established against Candida albicans (Tabbene et al., 2016).

Along with synthetic substances, natural antimicrobial peptides also have pronounced antimicrobial activity. For example, NA-CATH cathelicidin derived from the Chinese cobra, or mouse cathelicidin (mCRAMP) or human cathelicidin LL-37, which, even at low micromolar concentrations, is effective against Mycobacterium smegmatis. Peptides LL-37 and mCRAMP showed synergism with rifampicin in MIC (minimum inhibitory concentration) assays, and polymyxin B showed synergism with LL37. For intracellular destruction of mycobacteria contained within macrophages, polymyxin B with LL-37 and rifampicin with LL-37 and mCRAMP have synergistic effects. Other authors have studied the combined effect of azithromycin and the antimicrobial peptide of cathelicidin LL-37 on gram-negative microorganisms resistant to many antibacterial preparations Pseudomonas aeruginosa, Klebsiella pneumoniae and Acinetobacter baumannii. Increased pharmaceutical activity of azithromycin has been demonstrated and its synergism in combination with LL-37 established (Lin et al., 2015). Research data have shown that biologically active substances of natural genesis are promising for combined use with antimicrobials in the fight against infectious diseases.

As of today it is relevant to implement alternative methods of treatment of infections caused by different microorganisms and to develop combined approaches for the treatment of infectious diseases (Lewies et al., 2018; Xu et al., 2018; Pizzolato-Cezar et al., 2019). Biologically active substances of probiotic origin, as alternative methods for the treatment of infectious diseases, are taking leading positions. They have several advantages: they are highly active, safe, have a broad spectrum of antimicrobial action, have low resistance rates, predominantly exhibit immunomodulatory properties, promote healing of infectious processes of different genesis and etiology (Berditsch et al., 2015; Thomsen et al., 2016; Isajenko et al., 2019). The combined approach is distinguished by the use of two or more therapies for the treatment of infectious diseases. Its advantage is the ability to overcome the individual limitations of each active substance (Mulani et al., 2019). Even when the two inhibitors do not have to interact synergistically and this is scientifically proven, there are exceptions. Dillon et al. (2019) have proven the ability of a drug to supplement the kinetic disadvantages of another by accelerating initiation and increasing the duration of translation inhibition. The efficacy of combination therapy of two incompatible drugs has been confirmed in a model of pneumonia caused by the polyresistant strain of $A$. baumannii, due to a decrease in bacterial load on the lungs and an increase in survival (Dillon et al., 2019).

Products of probiotic strains of microorganisms are attractive to scientists as combination therapy with production antibacterial agents. The joint action of biologically active substances of probiotic microorganisms with antibiotics can increase the antimicrobial activity of industrial preparations, reduce the required concentration of the antibiotic, and therefore the likelihood of the development of resistance. These benefits of combined use can give hope for the future of extending the service existing antimicrobials.

The objective of this study was to substantiate the possibility developing "accompaniment-preparations" for combination therapy of infectious diseases of different genesis and etiology based on the metabolite complexes of L. rhamnosus GG and S. boulardii, which potentiate antibacterials and increase the sensitivity of Corynebacterium spp. to their joint action.

\section{Materials and methods}

As producers of structural components we used Lactobacillus rhamnosus GG from PREEMA ${ }^{\circledR}$ symbiotics (Schonen, Switzerland) and Saccharomyces boulardii from BULARDI ${ }^{\circledR}$ probiotic preparation (Schonen, Switzerland).

The lyophilizates of the microorganisms were suspended in isotonic solution of sodium chloride $(0.9 \%)$. To obtain the microbial masses of probiotic strains of lactobacteria and saccharomycetes, the material was subcultivated in a regulated nutrient medium for 20-24 hours at $37 \pm 1^{\circ} \mathrm{C}$. The purity of cultures of Lactobacillus bacteria and Saccharomyces fungi was confirmed by microscopic, bacteriological methods. The resulting microbial mass was washed three times from with medium. Suspensions of cells of microorganisms with an optical density of 10.0 units on the McFarland scale were prepared using the appliance Densi-La-Meter (PLIVA-Lachema Diagnostika, Czech Republic).

From the production cultures of bacteria and fungi, disintegrates were obtained (structural components). The disintegration of cell suspensions of probiotic strains was performed using a low-frequency generator G3-109 loaded on circular piezoelectric convertors, in a sparing mode of exposure (Isajenko et al., 2017; Isajenko et al., 2018).

Obtaining metabolites (structural-metabolites substances) of bacteria and fungi in disintegrates of probiotic cultures: sample ML-1 and ML-10 - metabolites of Lactobacillus were obtained as a result of cultivation of suspensions of $L$. rhamnosus GG with optical density of 1.0 and 10.0 units according to McF scale, respectively, in their own disintegrates (Isajenko et al., 2019), sample MS-1 and MS-10 - metabolites of Saccharomyces were obtained by growing a suspension of $S$. boulardii with optical density of 1.0 and 10.0 units according to McF scale, respectively, in the mushroom's own disintegrates, sample LS - metabolites of saccharomyces, different from the previous ones, were obtained by cultivation of mushroom suspensions in disintegrates of L. rhamnosus GG; sample MLS-1 and MLS-10 - a combination of metabolites of Lactobacillus and Saccharomyces, was obtained by the joint cultivation of microbial cells of L. rhamnosus GG and S. boulardii with optical density of 1.0 and 10.0 units according to McF scale, respectively, in disintegrates of lactobacteria (Isajenko et al., 2018).

Obtaining metabolites of bacteria and fungi in a liquid nutrient medium: sample ML-1 (b) and ML-10 (b) - metabolites of Lactobacillus, was obtained as a result of cultivation of suspensions of $L$. rhamnosus GG with optical density of 1.0 and 10.0 units according to McF scale, respectively, in a liquid nutrient medium, in particular, in nutrient broth with the addition of $1 \%$ glucose (Isajenko et al., 2019); sample MS-1 (b) and MS-10 (b) - metabolites of Saccharomyces, was obtained as a result of cultivation of suspensions of $S$. boulardii with optical density of 1.0 and 10.0 units according to McF scale, respectively, in a liquid nutrient medium, in particular, in nutrient broth with the addition of $1 \%$ 
glucose; sample MLS-1 (b) and MLS-10 (b) - a combination of metabolites of Lactobacillus and Saccharomyces, obtained by the joint cultivation of microbial cells of $L$. rhamnosus GG and $S$. boulardii with optical density of 1.0 and 10.0 units according to McF scale, respectively, in a liquid nutrient medium, in particular, in nutrient broth with the addition of 1\% glucose (Isajenko et al., 2019).

Ultrasonic disintegrates and cultures, grown in disintegrates and traditional culture medium were centrifuged at $1100 \mathrm{~g}$ for 15 minutes, then supernatants were filtered through sterile membrane filters with pore diameter equaling $0.2 \mu \mathrm{m}$ (Vladipor, Russia). Obtained filtrates of disintegrates (structural components) of L. rhamnosus GG - sample L, and S. boulardii-sample S.

The test-material of the filtrates of probiotic strains of microorganisms:

1) structural components of lactobacteria (L);

2) structural components of saccharomycetes $(\mathrm{S})$.

Metabolites, obtained by the author's method:

3) metabolite compounds of lactobacteria (ML), obtained by cultivation of lactobacteria in their own ultrasonic disintegrates;

4) metabolite compounds of saccharomycetes (MS), obtained by cultivation of sacchromycetes in their own ultrasonic disintegrates;

5) metabolite compounds of saccharomycetes, obtained by cultivation of sacchromycetes in ultrasonic disintegrates of lactobacteria (LS);

6) combination of metabolite compounds of lactobacteria and saccharomycetes, obtained by cultivation of producers $L$. rhamnosus GG and S. boulardii in ultrasonic disintegrates of lactobacteria (MLS);

Metabolites, obtained by traditional methods:

1) metabolite compounds of lactobacteria (ML (b)), obtained by cultivation of lactobacteria in nutrient broth with the addition of $1 \%$ glucose;

2) metabolite compounds of saccharomycetes (MS (b)), obtained by cultivation of sacchromycetes in nutrient broth with the addition of $1 \%$ glucose;

3) combination of metabolite compounds of lactobacteria and saccharomycetes, obtained by cultivation of producers $L$. rhamnosus $\mathrm{GG}$ and $S$. boulardii in nutrient broth with the addition of $1 \%$ glucose (MLS (b)).

At all stages of the development of remedies containing the metabolite substances and structural components of lactobacteria and saccharomycetes, the basic requirements that are imposed on the industrial conditions of production of probiotics and preparations developed on their basis were maximally met. Microbiological purity of intermediate and final test substances in the absence of extraneous microorganisms was carried out at each stage of the study.

The antimicrobial activity of the combined use of filtrates of L. rhamnosus GG and $S$. boulardii with antibacterial agents and the determination of the susceptibility of microorganisms to their joint action was performed on test-strains of Corynebacterium spp. tox ${ }^{+}$. For the experiments, daily cultures of bacteria grown on regulated nutrient media were used to meet the needs of the microorganisms (Atlas, 2010). Preparation of nutrient media was carried out in accordance with the requirements of the manufacturer. Their quality control was carried out according to the recommendations of the manufacturing companies, which are set out in the certificates for products, as well as the information sheet (Information sheet of the Ministry of Health of Ukraine No 05.4.1/1670 "Bacteriological control of nutrient media", Kiev, 2000). Microbial suspensions were prepared using isotonic solution of sodium chloride $(0.9 \%)$. The optical density of the samples corresponded to 0.5 units and 5.0 units on the McFarland scale (Densi-La-Meter, PLIVALachema Diagnostika, Czech Republic). Synchronization of the test cultures was performed under hypothermic conditions at $4 \pm 1^{\circ} \mathrm{C}$.

Investigation of the effect of filtrates of ultrasound disintegrates and metabolites of probiotic strains of lactobacteria and saccharomycetes, obtained in their own ultrasound disintegrates and nutrient broth with the addition of $1 \%$ glucose, on susceptibility of strains of Corynebacterium $\mathrm{spp}$. to antibacterial preparations.

Suspensions of test-cultures with optical density of 5.0 units according to $\mathrm{McF}$ scale were added to the filtrate of probiotic microorganisms, (test samples) or to the isotonic solution of sodium chloride $(0.9 \%)$ (control samples) in a ratio $1: 1$. The test and control samples were kept at $35 \pm 1{ }^{\circ} \mathrm{C}$ for 1 hour. After exposure, the optical density of the suspensions' test-culture of the test and control samples was brought to 0.5 units on the McF scale according to the guidelines (MOZ, 2007). The sowing of the material was carried out onto solid medium of Mueller Hinton. Standard discs with antibiotics were applied to the sown surface of the medium. They were incubated at $35 \pm 1^{\circ} \mathrm{C}$ for 24 hours. The results were recorded by measuring the growth retardation zones of the cultures around the discs with antimicrobial preparations.

In the study of the effect of filtrates of disintegrates and metabolites of probiotic strains of microorganisms on the susceptibility of test-cultures to antibiotics, we used one representative from each group of antimicrobial agents. Formation of a set of antibacterials for the evaluation of susceptibility of corynebacteria was performed according to the methodological recommendations (MOZ, 2007; Volyanskiy et al., 2014). Thus, in determining the sensitivity of toxigenic representatives of the genus Corynebacterium, after their pre-incubation with the test substances, to antibiotics from the group of carbapenems we investigated imipenem, from glycopeptides - vancomycin, from cephalosporins cefotaxime, from aminoglycosides - gentamicin, from macrolides erythromycin, from the quinolones - ciprofloxacin.

To determine the effectiveness of the joint use of antibiotics with the structural components and metabolites of lactobacteria and saccharomycetes, we conducted preliminary preparation of the discs. Standard discs with antibacterial preparations were kept in the test filtrates for 1 hour at $37 \pm 1{ }^{\circ} \mathrm{C}$ for maximum absorption. As a positive control we used a disc with antibiotics, a negative - a disc with sterile isotonic solution of sodium chloride ( $0.9 \%$ ). Prepared experimental and control discs were applied to the nutrient medium with crops of test strains of microorganisms. To ensure diffusion, they were previously kept at $4 \pm 1{ }^{\circ} \mathrm{C}$ for 1 hour. They were then incubated at $37 \pm 1^{\circ} \mathrm{C}$ for 24 hours and the zone of inhibition of growth of the microorganisms was measured. Comparisons were made with respect to control discs with antibacterial preparations.

The results of the studies were processed using Statistica 8.0 (StatSoft Inc., USA). The arithmetic mean (x) and standard error of the arithmetic mean (SD) were calculated. The reliability of the differences between the obtained data was determined using the non-parametric Ucriterion of Mann-Whitney. The difference between the test samples and the control samples was taken into account at the values $\mathrm{P}<0.05$.

\section{Results}

The results of growth retardation zones of Corynebacterium spp. tox ${ }^{+}$ to antibacterials after exposure to experimental filtrates of $L$. rhamnosus GG and $S$. boulardii, obtained by cultivation of the producers in ultrasound disintegrates and nutrient broth with the addition of $1 \%$ glucose (Fig. 1-4), showed different results. Determination of susceptibility of strains Corynebacterium spp. tox ${ }^{+}$after application of disintegrates and metabolites of probiotic strains of lactobacteria and saccharomycetes, obtained by the author's and traditional methods to glycopeptide antibiotics, in particular vancomycin, showed an increase in the zones of inhibition of visible growth of pathogens. Treatment of cells of toxigenic strains of diphtheria bacteria with structural components and metabolite compounds of $S$. boulardii resulted in a lesser increase in the sensitivity of corynebacteria compared with the filtrates of $L$. rhamnosus GG. The probable difference in the effect of test substances of saccharomyces with respect to pathogenic representatives of the genus Corynebacterium relative to the control samples was not observed regardless of the method of obtaining metabolites and the sowing dose of the probiotic strain.

Authentic increase in the sensitivity of corynebacteria to vancomycin was observed after exposure with all filtrates of lactobacteria. The differences in the results of the study of the effect of structural components of lactobacilli, metabolites of lactobacilli, a mixture of metabolites of saccharomyces and lactobacteria on pathogenic bacteria, were observed depending on the initial concentration of L. rhamnosus in the culture obtained by our own and traditional methods. Thus, the use of high inoculating doses of lactobacteria increased the zones of inhibition of visible growth of corynebacteria more (by 12.0, 9.6, 4.0, and $1.6 \mathrm{~mm}$ ) than using smaller initial doses (by 7.4, 6.25, 2.2, and $1.2 \mathrm{~mm}$ ). An increase of susceptibility of strains Corynebacterium spp. tox ${ }^{+}$was observed regardless of the mode of production of metabolites $L$. rhamnosus. 

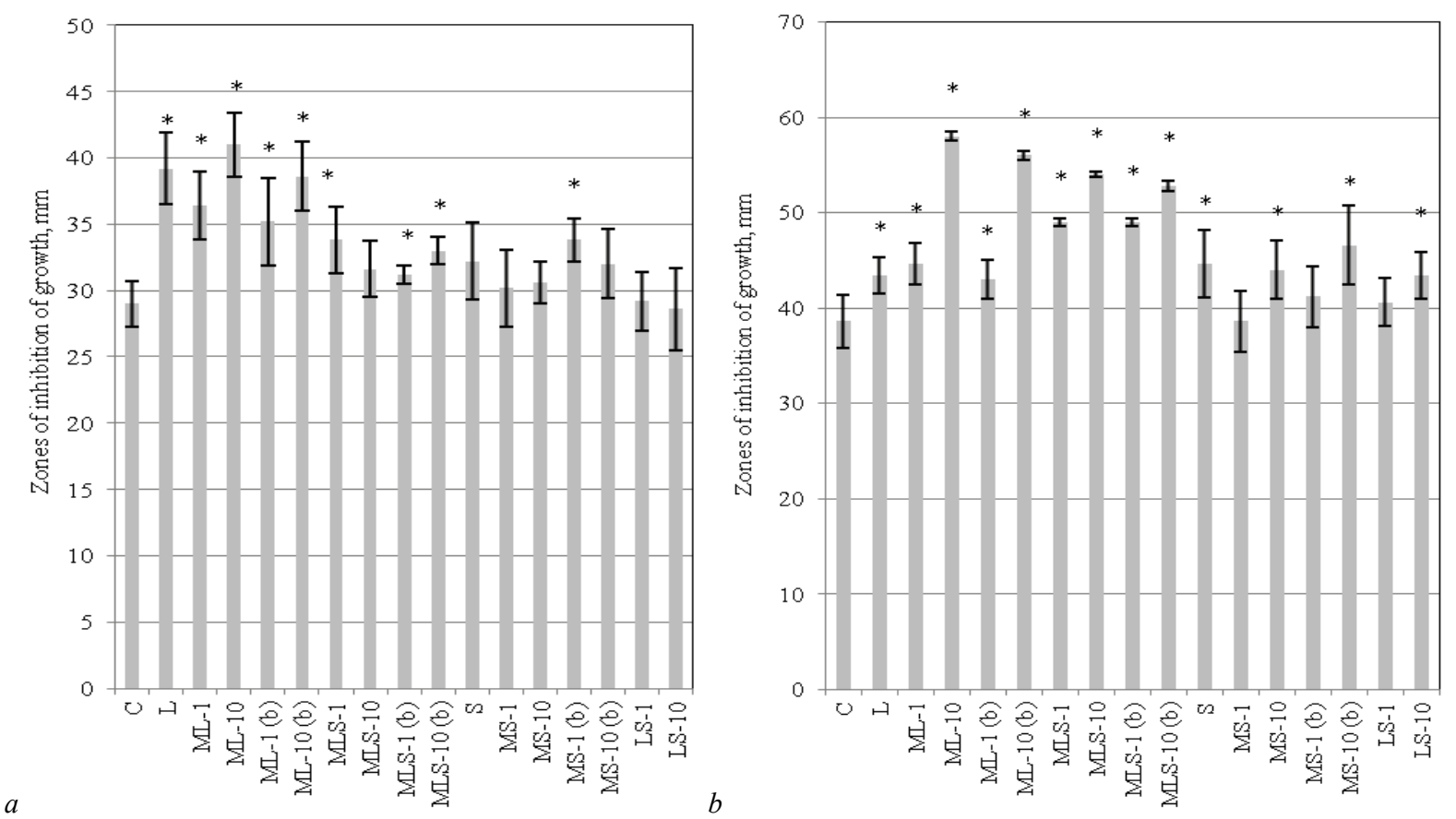

Fig. 1. Diameters of inhibition zones of growth Corynebacterium spp. tox ${ }^{+}$to antibacterials preparations (vancomycin $(a)$, penicillin $(b)$, after exposure to the filtrates (structural components and metabolites) of probiotic strains of Lactobacillus rhamnosus GG and Saccharomyces boulardii obtained by growing the producers in their own ultrasonic disintegrates and nutrient broth with the addition of $1 \%$ glucose $(\mathrm{CU}, \mathrm{x} \pm \mathrm{SD}, \mathrm{n}=5)$ : $\mathrm{C}$ - control (isotonic solution of sodium chloride $(0.9 \%), \mathrm{L}$ - filtrates of disintegrates (structural components) of L. rhamnosus GG, ML-1 and ML-10 - metabolites (metabolites' compounds) of lactobacteria obtained by growing the producers (with optical density, corresponding to 1.0 and 10.0 units on the McFarland scale, respectively) in their own ultrasonic disintegrates, ML-1 (b) and ML-10 (b) - metabolites (metabolites' compounds) of lactobacteria, obtained by growing the producers (with an optical density, corresponding to 1.0 and 10.0 units on the McFarland scale, respectively) in nutrient broth with the addition of $1 \%$ glucose, MLS- 1 and MLS- $10-$ a combination of metabolites of lactobacteria and saccharomycetes, obtained by growing producers (with an optical density corresponding to 1.0 and 10.0 units on the McFarland scale, respectively) in ultrasonic disintegrates of lactobacteria, MLS-1 (b) and MLS-10 (b) - a combination of metabolites of lactobacteria and saccharomycetes, obtained by growing producers (with an optical density corresponding to 1.0 and $10.0 \mathrm{McFarland}$ units, respectively) in nutrient broth with the addition of $1 \%$ glucose, $\mathrm{S}$ - filtrates of disintegrates (structural components) of S. boulardii, MS-1 and MS-10 - metabolites (metabolites' compounds) of saccharomycetes obtained by growing the producers (with optical density, corresponding to 1.0 and 10.0 units on the McFarland scale, respectively) in their own ultrasonic disintegrates, MS -1 (b) and MS-10 (b) - metabolites (metabolites' compounds) of saccharomycetes obtained by growing the producers (with an optical density, corresponding to 1.0 and 10.0 units on the McFarland scale, respectively) in nutrient broth with the addition of $1 \%$ glucose, LS- 1 and

LS-10 - metabolites (metabolites compounds) of saccharomycetes obtained by growing the producers (with optical density, corresponding to 1.0 and 10.0 units on the McFarland scale, respectively) in ultrasonic disintegrates of lactobacteria; * - the difference between the experimental samples and the control samples was statistically significant $(\mathrm{P}<0.05)$

The maximum increase in the growth retardation zones of corynebacteria for vancomycin was established after incubation with the filtrates of disintegrates and metabolites, obtained by both methods (by 6.25 $12.0 \mathrm{~mm}$ ) (Fig. 1). The results of the study of the effect of a mixture of metabolites of saccharomycetes and of lactobacteria on the sensitivity of pathogenic representatives of the genus Corynebacterium were close to the results of the study of the influence of the structural components and metabolites of saccharomycetes, obtained by the cultivation of producers in disintegrates and nutrient broth with the addition of $1 \%$ glucose.

Penicillins, carbapenems and cephalosporins by their mechanism of action, as well glycopeptide antimicrobial preparations, violate the synthesis of the cell wall of bacteria. The use of disintegrates and metabolites of lactobacteria and saccharomycetes, obtained by the two presented methods, on the toxigenic strains of corynebacteria showed an increase in the sensitivity of pathogenic microorganisms.

After pre-exposure of Corynebacterium spp. tox ${ }^{+}$in filtrates of lactobacteria and saccharomycetes, their sensitivity to penicillin increased regardless of the method of production (Fig. 1). The increase in the diameter of the growth retardation zone of pathogenic microorganisms depended on the initial concentration of cells of L. rhamnosus and S. boulardii in the initial suspension of the producers. The effect of the filtrates with higher initial doses of probiotic microorganisms (with optical density of 10.0 units according to $\mathrm{McF}$ scale) on the Corynebacterium led to a higher sensitivity of the toxigenic Corynebacterium strains (by 6.0
$19.4 \mathrm{~mm}$ ) compared to the use of lower initial concentrations of probiotics (with optical density of 1.0 units according to McF scale) (by 4.6$10.3 \mathrm{~mm}$ ). The results obtained are consistent with those of the susceptibility study of corynebacteria to vancomycin. Treatment with a combination of metabolites of lactobacteria and saccharomyces was accompanied by a significant increase in the sensitivity of bacteria to penicillins (by 14.6-15.4 mm). The maximum increase in the growth retardation zones of the test-strains in response to the penicillin, as well as to the vancomycin, was observed after incubation of Corynebacterium cells with filtrate metabolites of lactobacteria, obtained by both methods, using high starting concentrations of producer (by 17.4-19.4 mm, $\mathrm{P}=0.001$ ). The influence of disintegrates and metabolites of saccharomyces on pathogenic representatives of the genus Corynebacterium led to increased sensitivity of microorganisms to penicillin. Among the filtrates of S. boulardii, the sensitivity of the toxigenic strains (by 4.7 to $8.0 \mathrm{~mm}$ ) was statistically significantly increased compared to the control $(\mathrm{P}<0.05)$ samples with higher seeding concentration of probiotic cells, regardless of the method of production. Research on response of suspensions of microbial cells of Corynebacterium spp. tox ${ }^{+}$of different cultures to cephalosporins, in particular to cefotaxime, showed their high sensitivity in control samples $(37.2 \pm 2.7 \mathrm{~mm}$, Fig. 2). A statistically significant increase in the diameter of the growth retardation zones of corynebacteria was observed after their pre-incubation with the structural components and metabolites of $L$. rhamnosus (by $2.8-9.5 \mathrm{~mm}, \mathrm{P}=0.04$ ). 

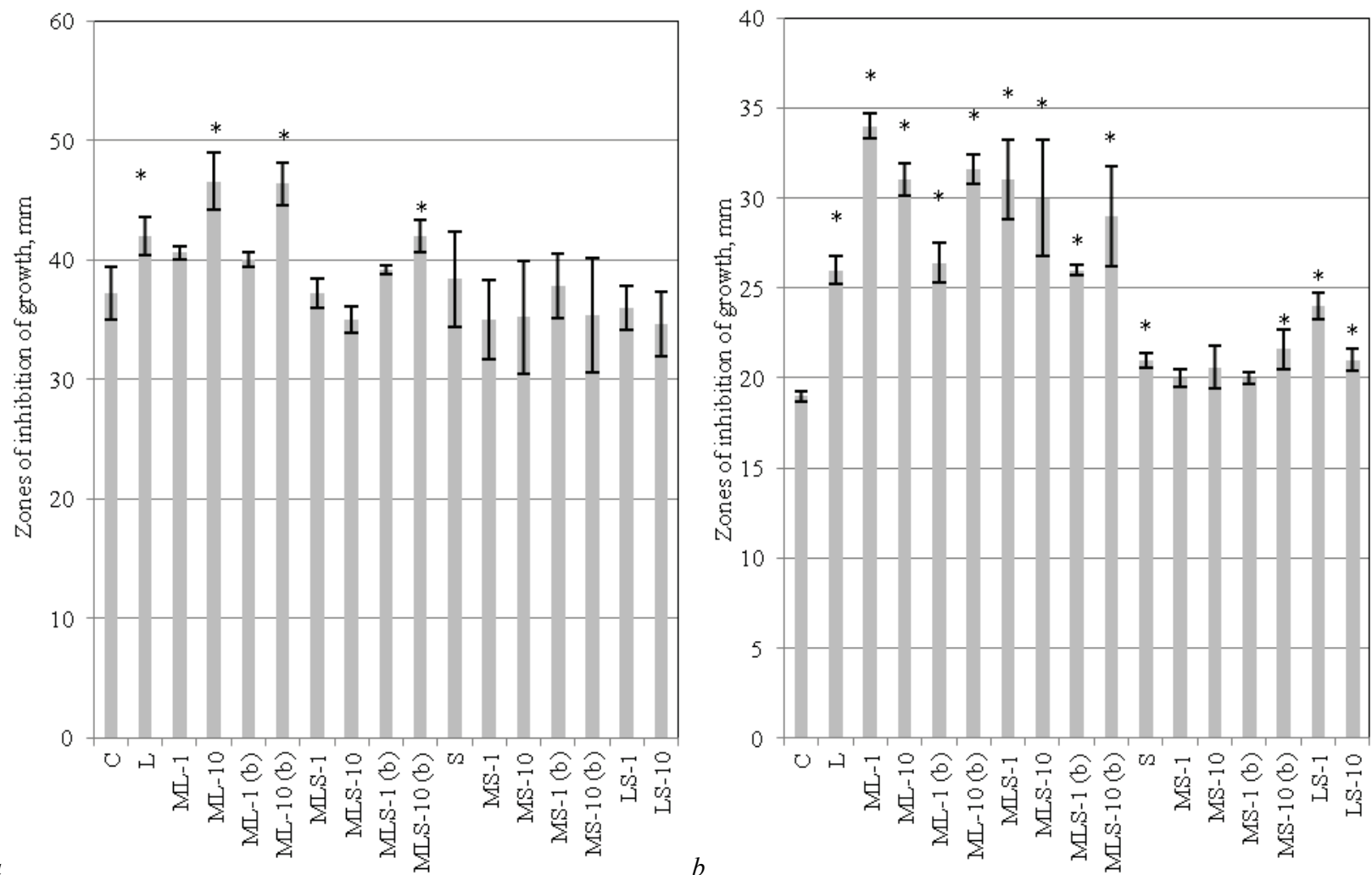

Fig. 2. Diameters of inhibition zones of growth of Corynebacterium spp. tox ${ }^{+}$to antibacterial preparations (cefotaxime $(a)$, imipenem $(b)$ ), after exposure to the filtrates (structural components and metabolites) of probiotic strains of Lactobacillus rhamnosus GG and Saccharomyces boulardii obtained by growing the producers in their own ultrasonic disintegrates and nutrient broth with the addition of $1 \%$ glucose $(C U, x \pm S D, n=5)$ : see Fig. 1
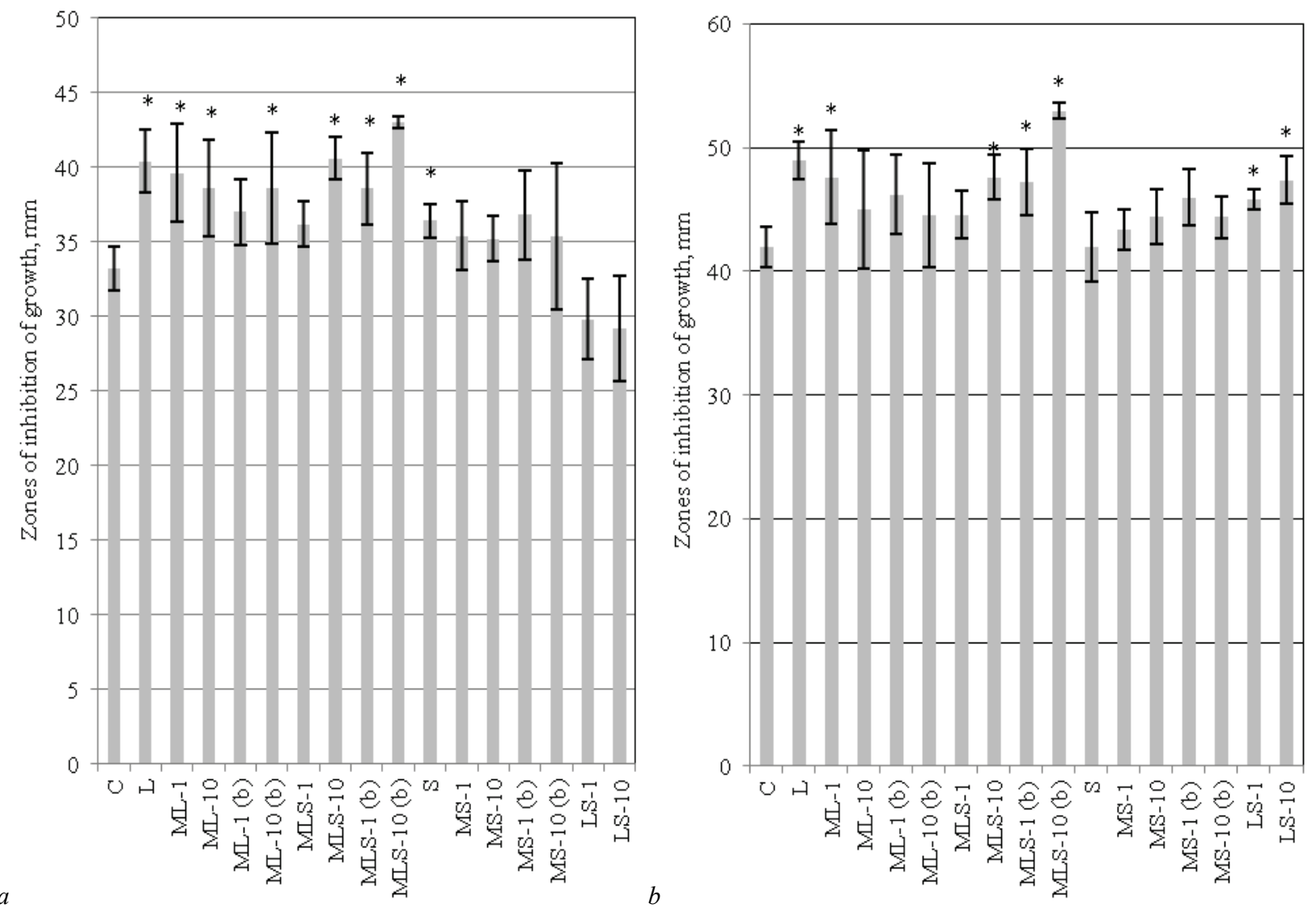

Fig. 3. Diameters of inhibition zones of growth of Corynebacterium spp. tox ${ }^{+}$to antibacterials preparations (ciprofloxacin (a), gentamicin $(b)$ ), after exposure to the filtrates (structural components and metabolites) of probiotic strains of Lactobacillus rhamnosus GG and Saccharomyces boulardii obtained by growing the producers in their own ultrasonic disintegrates and nutrient broth with the addition of $1 \%$ glucose $(\mathrm{CU}, \mathrm{x} \pm \mathrm{SD}, \mathrm{n}=5)$ : see Fig. 1 

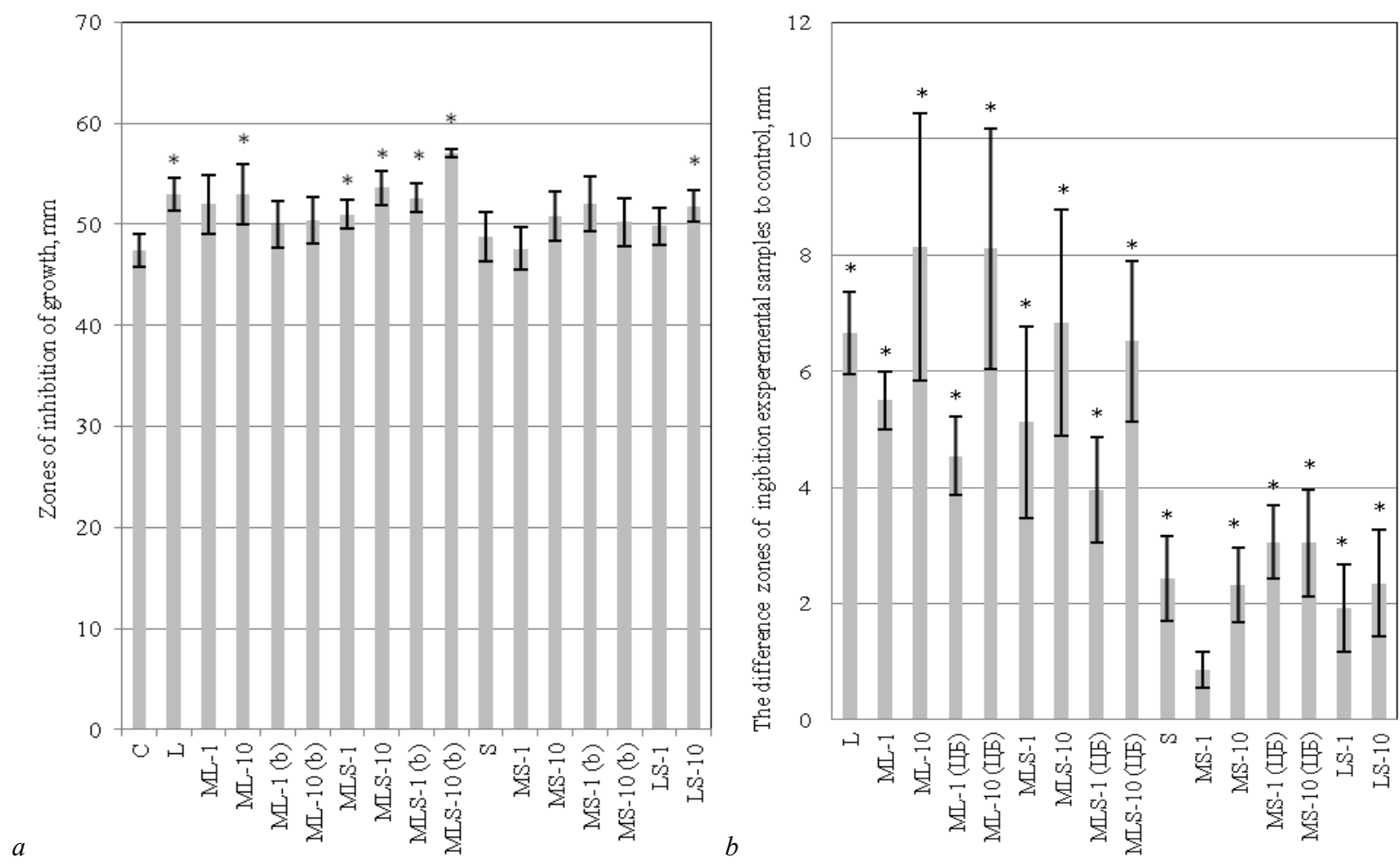

Fig. 4. Diameters of inhibition zones of growth of Corynebacterium spp. tox ${ }^{+}$to antibacterial preparations (erythromycin (a)), after exposure to the filtrates (structural components and metabolites) of probiotic strains of Lactobacillus rhamnosus GG and Saccharomyces boulardii obtained by growing the producers in their own ultrasonic disintegrates and nutrient broth with the addition of $1 \%$ glucose and comparing the effectiveness of the filtrates in increasing growth retardation zones of Corynebacterium spp. tox ${ }^{+}$of experimental samples to the control samples in different groups of antibacterial agents (imipenem, vancomycin, cefotaxime, gentamicin, erythromycin, ciprofloxacin, penicillin) $(b)(C U, x \pm S D, n=5)$ : see Fig. 1

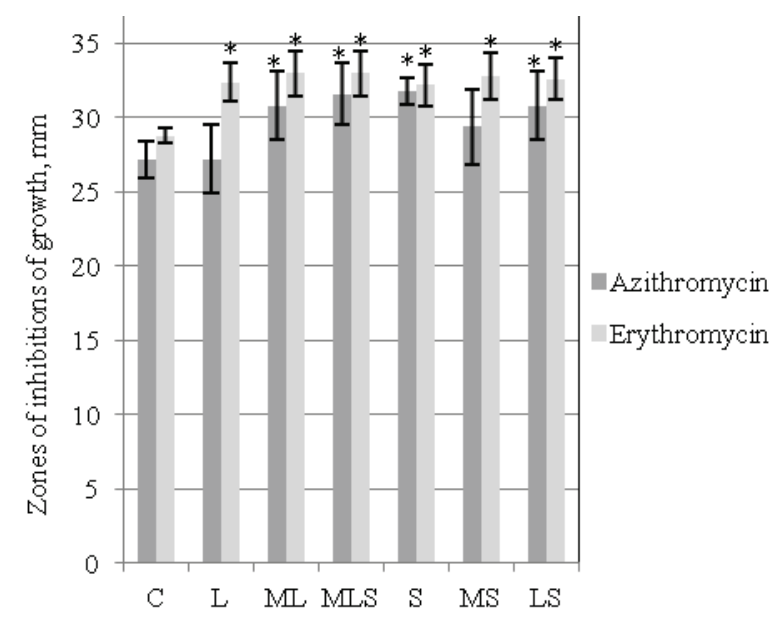

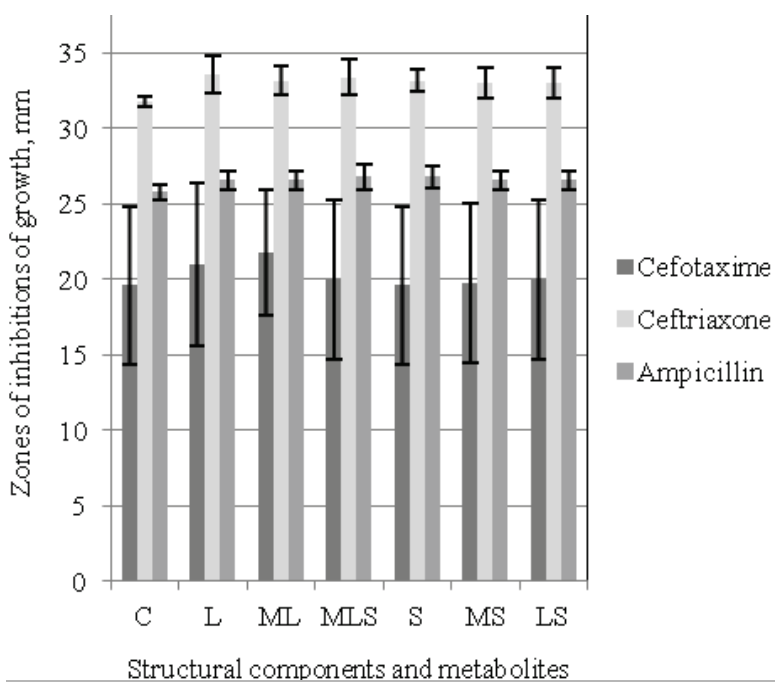

Fig. 5. Diameters of inhibition zones of growth of Corynebacterium spp. tox ${ }^{+}$to the co-administration of antibiotics (azithromycin, erythromycin $(a)$, cefotaxime, ceftriaxone, ampicillin $(b)$ ) with the filtrates (structural components and metabolites) of probiotic strains of Lactobacillus rhamnosus GG and Saccharomyces boulardii obtained by growing the producers in their own ultrasonic disintegrates $(\mathrm{CU}, \mathrm{x} \pm \mathrm{SD}, \mathrm{n}=5): \mathrm{C}$ - control (isotonic solution of sodium chloride $(0.9 \%)), \mathrm{L}$ - filtrates of disintegrates (structural components) of L. rhamnosus GG, ML - metabolites (metabolites' compounds) of lactobacteria, obtained by growing the producers in their own ultrasonic disintegrates, MLS - a combination of metabolites lactobacteria and saccharomycetes, obtained by growing producers in ultrasonic disintegrates of lactobacteria, $\mathrm{S}$ - filtrates of disintegrates (structural components) of S. boulardii, MS - metabolites

(metabolites' compounds) of saccharomycetes, obtained by growing the producers in their own ultrasonic disintegrates,

LS - metabolites (metabolites' compounds) of saccharomycetes, obtained by growing the producers in ultrasonic disintegrates of lactobacteria; * - the difference between the experimental samples and the control samples was statistically significant $(\mathrm{P}<0.05)$

The results on the impact of the researched samples on the enhancement of the sensitivity of the toxigenic strains against cefotaxime confirmed our previous data. The maximum increase of the zones of inhibition of growth of pathogenic microorganisms was established after incubation of cells with metabolites of lactobacteria, obtained by both methods, when using high initial concentrations of probiotic. Exposure to structural components, metabolites of saccharomycetes, and a combination of metabolites of saccharomycetes and lactobacteria did not significantly increase the sensitivity of pathogenic representatives of the genus Corynebacterium to the antibacterial preparation of the cephalosporin series. 
Determination of the susceptibility of microbial suspensions of $\mathrm{Co}$ rynebacterium spp. tox ${ }^{+}$showed the resistance of the selected strains to preparations of carbapenems (imipenem) in the control samples (Fig. 2). According to the results of exposure to the toxigenic corynebacteria of all test substances of lactobacteria, regardless of the conditions of their cultivation and the optical density of the producer, there was a statistically significant increase in the zones of inhibition of the growth of pathogenic microorganisms. The highest rates of sensitivity increase were obtained after incubation of pathogens with filtrates of culture lactobacteria with high initial optical density, regardless of the cultivation method (by $12.5-15.0 \mathrm{~mm})(\mathrm{P}<0.05)$. No significant differences were found between the increase in susceptibility of corynebacteria after exposure to the structural components and metabolites of saccharomycetes obtained by our own and traditional methods.

The results of the studies showed that after pre-incubation of strains of Corynebacterium spp. tox ${ }^{+}$in filtrates of culture lactobacteria with high initial optical density obtained by growing the producers in their own disintegrates and nutrient broth with the addition of $1 \%$ glucose, sensitivity increased to the similar mechanism of action of penicillins, carbapenems, cephalosporins and glycopeptides. And after incubation of the test-cultures with all the test substances of saccharomyces, regardless of the conditions of cultivation and the optical density of the producers, there was no increase in sensitivity or an unreliable increase of zones of inhibition of growth of pathogenic microorganisms.

The study of the influence of structural components and metabolites of lactobacteria and saccharomycetes, obtained by cultivation of the producer in disintegrates or nutrient broth with the addition of $1 \%$ glucose, on the sensitivity of toxigenic corynebacteria to quinolones (ciprofloxacin), which by the mechanism of action inhibit bacterial synthesis DNA, presented in Figure 3, showed different results. Thus, all test substances of saccharomycetes, with high and low initial optical densities and different cultivation conditions, did not statistically significantly increase the sensitivity of strains Corynebacterium spp. tox ${ }^{+}$to quinolones preparations $(\mathrm{P}<0.05)$. Structural components and metabolites of lactobacteria increased the sensitivity of corynebacteria to ciprofloxacin. We found a less pronounced antimicrobial effect compared to imipenem, vancomycin, cefotaxime and penicillin. The maximum increase in the zones of inhibition of growth of pathogens was observed after incubation of test cells with disintegrates of lactobacteria, metabolites of lactobacteria and a combination of metabolites of lactobacteria and saccharomycetes obtained by both methods at the application of high initial concentrations of probiotics (by $3.0-9.8 \mathrm{~mm}$ ). According to the results obtained, the maximum increase in the sensitivity of toxigenic corynebacteria was observed after their incubation in combination with metabolites of probiotic strains of lactobacteria and saccharomycetes obtained by cultivation of the producers in their own ultrasonic disintegrates or nutrient broth with the addition of $1 \%$ glucose (by 7.3-9.8 mm).

The effect of structural components and metabolites of lactobacteria and saccharomycetes on the sensitivity of corynebacteria to antibacterial preparations that inhibit bacterial protein synthesis - macrolides (erythromycin) and aminoglycosides (gentamicin), showed similar results (Fig. 3, 4). Thus, the increase in the sensitivity of toxigenic representatives of the genus Corynebacterium, after incubation in all experimental filtrates of saccharomycetes, with low and high initial optical density, obtained by the author's and traditional methods, was less pronounced (by 0.0 $5.4 \mathrm{~mm}$ ) for aminoglycosides and (by $1.4-4.4 \mathrm{~mm}$ ) for macrolides.

The increase in the sensitivity of pathogens was observed after preexposure of microbial cells with structural components of lactobacteria, metabolites of lactobacteria and a combination of metabolites of lactobacteria and saccharomycetes, regardless of the conditions of cultivation and optical density of the producers (by $2.0-11.0 \mathrm{~mm}$ ) for the aminoglycosides and (by 2.6-9.6 mm) for the macrolides. The maximum increase in the zones of inhibition of corynebacteria growth was observed after incubation of cells of pathogenic microorganisms with a combination of metabolites of lactobacteria and saccharomycetes with a high sowing dose of producers (by 11.0 and $9.6 \mathrm{~mm}$ for aminoglycosides and macrolides respectively). Compared to previous studies, the increased sensitivity of Corynebacterium to macrolides and aminoglycosides also depended on the optical density of the producers. Comparative determination of the most effective biologically active substance among all experimental samples of L. rhamnosus GG, S. boulardii obtained by cultivation of producers in disintegrates or nutrient broth with the addition of $1 \%$ glucose, to increase the sensitivity of strains Corynebacterium spp. tox ${ }^{+}$to different groups of antibacterials is presented in Figure 4. The maximum statistically significant increase in the zones of inhibition of growth of corynebacteria with respect to the selected antibacterial drugs was observed with the use of metabolites of lactobacteria with high initial optical density of the producers, obtained by our own and traditional methods (by $8.1 \mathrm{~mm}, \mathrm{P}=0.003$ ). A statistically significant increase in the sensitivity of Corynebacterium spp. tox ${ }^{+}$was also observed after exposure to ultrasonic disintegrates of lactobacteria and combinations of metabolites of probiotic strains of lactobacteria and saccharomycetes with a high sowing dose of the producer, irrespective of the production method (by 5.1-6.6 mm). Minimal increase in the zones of inhibition of growth of corynebacteria was obtained when applying the filtrates of disintegrates and metabolites of saccharomycetes, regardless of the conditions of cultivation and the optical density of the producers (1.9-3.0 mm). Therefore, all presented biologically active substances of lactobacteria and saccharomycetes to varying degrees increase the sensitivity of strains of Corynebacterium spp. tox ${ }^{+}$to antibiotics. The above research results give grounds to consider the developed probiotic substances as promising components of adjunctive antidiphtheria therapy.

The results obtained at the first stage of the work made it possible to conclude that, thanks to the cultivation of producers in disintegrates of probiotic microorganisms, metabolites were obtained with activity that is not inferior to the products of vital activity obtained by the cultivation of probiotic microorganisms in the traditional nutrient broth. Therefore, in the second stage of the study, we investigated the effect of structural components and metabolites of $L$. rhamnosus GG and $S$. boulardii, obtained by cultivation of producers, with an optical density corresponding to 10.0 units on the McFarland scale, in their own disintegrates, on the antimicrobial activity of antibacterial preparations. A study of the synergistic interaction of test filtrates with antibacterial preparations against strains of Corynebacterium spp. tox ${ }^{+}$was performed on macrolides (azithromycin, erythromycin) and beta-lactams (cefotaxime, ceftriaxone, ampicillin) (Fig. 5). The combined use of substances L. rhamnosus GG and $S$. boulardii with azithromycin was accompanied by a statistically significant increase in the zones of inhibition of the growth of toxigenic strains of corynebacteria (by $2.4-4.6 \mathrm{~mm}$ ). The maximal increase in the antimicrobial activity of azithromycin was observed under the influence of metabolites of lactobacteria and the combination of metabolites of lactobacteria and saccharomycetes by 4.6 and $4.4 \mathrm{~mm}$, respectively $(\mathrm{P}=0.02)$.

Joint application of the structural components and metabolites of the probiotic strains of L. rhamnosus GG and S. boulardii with erythromycin showed similar results. The increase of erythromycin occurred under the influence of all filtrates of disintegrates and products of metabolites of lactobacteria and saccharomycetes: the diameter of the growth retardation zone of Corynebacterium increased by $4.2-4.5 \mathrm{~mm}$. The maximal increase of the zones of inhibition of growth of corynebacteria by $4.5 \mathrm{~mm}$ occurred under the influence of metabolites of lactobacteria and the combination of metabolites of saccharomycetes and lactobacteria $(P=0.02)$. Combined use of azithromycin or erythromycin with experimental filtrates of $L$. rhamnosus $\mathrm{GG}$ and $S$. boulardii obtained the same results of potentiation of antimicrobial activity of both antibacterial preparations from the macrolide group.

The results of the study of the joint effect of the structural components and metabolites of probiotic strains of lactobacteria and saccharomycetes with cefotaxime, ceftriaxone, ampicillin on the toxigenic corynebacteria, which are presented in Figure 5, showed an increase in the antimicrobial action of the antibiotic of tested substances against Corynebacterium spp. tox ${ }^{+}$regardless of the combination of their usage. However, there was no statistically significant increase in growth retardation zones. Joint application of filtrates of L. rhamnosus GG and $S$. boulardii with cefotaxime increased the corynebacterium growth inhibition zones by $0.2-2.2 \mathrm{~mm}$, ceftriaxone by $1.2-1.8 \mathrm{~mm}$, and ampicillin by $0.8-1.0 \mathrm{~mm}$. The maximum increase in antimicrobial activity 
was established by the combination of cefotaxime $(2.2 \mathrm{~mm})$ with the products of vital activity of lactobacteria, and ceftriaxone (by $1.6 \mathrm{~mm}$ ) and ampicillin (by $1.0 \mathrm{~mm}$ ) with the combination of metabolites of lactobacteria and saccharomycetes.

When carrying out comparative evaluation of the most effective biologically active substance among the structural components of L. rhamnosus GG, S. boulardii, metabolites of lactobacteria and saccharomycetes, combinations of metabolites of probiotic strains of bacteria and fungi, obtained by cultivation of producers in disintegrates, with enhancement of antimicrobial activity of antibiotic preparations from different groups (azithromycin, erythromycin, cefotaxime, ceftriaxone, ampicillin), maximum increase in growth retardation zones of test samples of Corynebacterium spp. tox ${ }^{+}$relative to controls was established under the influence of metabolites of lactobacteria. These results are in line with increased sensitivity of Corynebacterium to the combined use of lactobacteria and saccharomycetes with antibiotics. Consequently, the metabolites of L. rhamnosus GG are the most active filtrate for potentiation of antimicrobial activity of antibacterial preparations and increase of susceptibility of corynebacteria.

\section{Discussion}

For research on the effect of the structural components and metabolites of probiotic strains of lactobacteria and saccharomycetes on the antimicrobial activity of antibiotics and the susceptibility of microorganisms to their joint action as test-cultures we selected Corynebacterium spp. tox ${ }^{+}$for the following reasons. The first reason. Diphtheria remains a real problem in the world today (WHO, 2007; MOZ, 2018; WHO, 2018). Venezuela, Indonesia, Yemen and Bangladesh requested the World Health Organization (WHO) to engage in anti-diphtheria measures during the diphtheria epidemic in 2017 (WHO, 2018). In two months 48 people died of diphtheria in Yemen alone, and the number of patients reached 678 (WHO, 2018). In 2018, WHO reported that diphtheria had become a real threat worldwide (WHO, 2018). Some cases of this disease have also been reported in Ukraine (MOZ, 2018). Therapy of severe forms of the disease, characterized by rapid flow and irreversible damage to many organs, requires additional treatment. The use of biologically active substances of lactobacteria and saccharomycetes for the development of "accompaniment-preparations" and their use as adjunctive therapy in the treatment of patients with diphtheria is likely to significantly reduce the duration of this pathology. This assumption is based on previously obtained data on the pronounced antimicrobial and antibiotic properties of the biologically active substances $L$. rhamnosus $\mathrm{GG}$ and $S$. boulardii against Corynebacterium spp. tox ${ }^{+}$and the results presented regarding the potentiation of the antimicrobial activity of antibacterials by the filtrates of L. rhamnosus GG and S. boulardii and increased sensitivity of Corynebacterium spp. to their combined application (Isayenko et al., 2019; Isajenko et al., 2019).

Second reason. The era of "post antibiotics" has begun. This is when even simple infectious diseases are not treatable due to a significant increase in the resistance of pathogens to antibacterial preparations (WHO, 2017; MOZ, 2018). The severity of the problem of the development of antibiotic resistance prompts doctors and scientists around the world to seek alternative treatments for infectious diseases (Chaudhary, 2016; Richardson et al., 2017; WHO, 2017; Pizzolato-Cezar et al., 2019) and to guide developments to reduce the likelihood of developing antibiotic resistance and reduce the number of resistant strains of microorganisms (WHO, 2018; Pizzolato-Cezar et al., 2019). It has been found that the effect of antibacterial preparations correlates with the formation of resistance in pathogens (Pizzolato-Cezar et al., 2019). Therefore, it is possible to reduce the development of resistance through more effective treatments for infections. This can be achieved, on the one hand, by increasing the sensitivity of pathogenic strains of microorganisms to existing antimicrobial preparations, as stated above. On the other hand, it can be achieved through the combined use of products of vital activity of probiotic origin with antimicrobial drugs for the treatment of infectious diseases. Studies on the interaction of antibiotics with lactobacteria and saccharomycetes in relation to pathogenic corynebacteria have not been performed previously. Influence of structural components and me- tabolites of $L$. rhamnosus GG and $S$. boulardii, obtained by cultivation of the producers in ultrasonic disintegrates, on the sensitivity of Corynebacterium spp. tox ${ }^{+}$to antibacterial preparations has also not been studied before.

The first area of research, presented in the paper, was on increase in the susceptibility of toxigenic strains of microorganisms to antimicrobial preparations, due to the consistent impact of products of lactobacteria and saccharomycetes and antibiotics on Corynebacterium spp. tox ${ }^{+}$. Initially, pathogenic bacteria were exposed to the structural components and metabolites of probiotic strains of $L$. rhamnosus GG and $S$. boulardii, obtained by cultivation of the producers in their own ultrasonic disintegrates and nutrient broth with the addition of $1 \%$ glucose. Then the sensitivity of Corynebacterium spp. tox ${ }^{+}$, previously placed in experimental biologically active substances, to antibacterial preparations was studied. The results of the study of the influence of the structural components and metabolites of probiotic strains of lactobacteria and saccharomycetes on the sensitivity of Corynebacterium spp. tox ${ }^{+}$with respect to antibacterial preparations, showed that all experimental filtrates increase the susceptibility of toxigenic corynebacteria to selected antibiotics. The differences between different groups of antibacterial preparations taken for the experiment were in the different mechanisms of their action on bacterial microbial cells. Thus, quinolones inhibit bacterial DNA synthesis, beta-lactams to which appertain penicillins, carbapenems and cephalosporins inhibit cell wall component biosynthesis, macrolides and aminoglycosides inhibit bacterial protein synthesis, and glycopeptides violate the synthesis of the cell wall of bacteria. When performing a comparative characterization of the effect of filtrates of L. rhamnosus GG and S. boulardii on the sensitivity of Corynebacterium spp. tox ${ }^{+}$to the different groups of antibacterial preparations, it was found that the change in the sensitivity of the microbial cells of the pathogen was different. The greatest inhibition of the diameter of the zone of growth retardation of pathogenic bacteria, after the use of biologically active substances of lactobacteria and saccharomycetes, was observed in relation to penicillins and carbapenems, then in relation to a representative of the glycopeptide antibiotic - vancomycin. The slightest increase in sensitivity of toxigenic representatives of the genus $\mathrm{Co}^{-}$ rynebacterium was observed for the preparations of quinolones, macrolides and aminoglycosides.

The results on varying levels of increase in sensitivity of pathogens to antibacterial preparations are explained by other researchers' data on the effects of biologically active substances on microbial cells (Cassone \& Otvos, 2010; Mardirossian et al., 2014; Reffuveille et al., 2014; Wenzel et al., 2014; Florin et al., 2017). This is attributed to differences in the different mechanisms of action of antimicrobial peptides in their effect on bacteria. The basic mechanism involves the incorporation of an antimicrobial substance into the bacterial membranes of the pathogenic object, destruction of membranes, leakage of cytoplasm and death of the microorganism. The complexity of the process can be considered at the stage of penetration of the biologically active substance through the membrane. It can occur in several ways. The most popular is the "poreforming" model, in which peptides interact with lipid bilayers embedded in the membrane, penetrating through it. The "barrel" method of membrane destruction involves the arrangement of peptide molecules perpendicular to the membrane. "Toroidal" mechanism is when the walls of the pores are composed of both peptide and lipid molecules - they tear down the entire membrane, it loses its stability, the content of the microbial cell spills out, the death of the pathogenic bacterium occurs. In the "carpet" model a molecular "carpet" is formed from positively charged peptide molecules and a negatively charged membrane of a microorganism. The rupture of the membrane into fragments occurs when the entire surface of the bacterium is occupied by peptides (Il'yashenko et al., 2012; Yasir et al., 2019). Secondary mechanisms of action of antimicrobial substances include metabolites' inhibition, inhibition of DNA, RNA and protein synthesis, induction of ribosomal aggregation, inhibition of septal formation, delocalization of membrane proteins, inhibition of cell wall synthesis, etc. (Ribeiro et al., 2015; Nagarajan et al., 2019). Therefore, the differences in the increase in the sensitivity of pathogenic microorganisms to antibacterial preparations can be explained by the different mechanism of action of biologically 
active substances on microbial cells. The peptide antibiotic klebsazolicin isolated from Klebsiella pneumoniae subsp. ozaenae suppresses the growth of sensitive cells by inhibiting protein synthesis due to binding to the bacterial ribosome (Metelev et al., 2017). The antimicrobial action of the sublancine produced by Bacillus subtilis 168 is manifested by the destruction of the bacterial cell wall. It also compensates the immune response during infection and relieves intestinal inflammation. Due to the combined antibacterial and immunomodulatory effect, researchers suggest its use in the treatment of resistant bacteria (Wang et al., 2017). Biologically active substances derived from Lactobacillus acidophilus exert an antimicrobial effect through direct inhibition of the growth of pathogenic microorganisms.

They also affect the cell wall of bacteria, production enzymes, which results in suppression of the virulence of the pathogen (Ismeeal et al., 2013; Satpute et al., 2016). Other antimicrobials increase bacterial sensitivity to antibiotics by reducing the number of outflow inhibitors (efflux pump inhibitors capable of expelling antibiotics from the cell) (Van Bambeke et al., 2006; George et al., 2011; Li et al., 2015; Ferrer-Espada et al., 2019). Through the interaction of peptides, which increase permeability, with the outflow inhibitors due to the reduction of their number, increase in the sensitivity of bacteria is achieved. Thus, the increased sensitivity of $P$. aeruginos $a$ to antibacterial drugs was established (Ferrer-Espada et al., 2019). These data are in good agreement with the presented results of our own research and prove the possibility of using biologically active substances to increase the sensitivity of bacteria to traditional antimicrobials. Despite the different mechanisms of action of antimicrobial peptides on pathogenic bacteria, their main advantage is the effect on the whole cellular organism rather than on a specific molecule.

In this study, it was found that among the research filtrates of L. rhamnosus GG and S. boulardii, the metabolites of lactobacteria with a high initial optical density of the microorganism possess the greatest ability to increase the sensitivity of corynebacteria to antibiotics. The results of varying degrees of increase in sensitivity of Corynebacterium spp. tox ${ }^{+}$to antibiotics, depending on the initial concentration of the producer used in the experiment, confirm the data of other authors. Studies conducted with pleurocidin have shown that at low inhibitory concentrations it has less potential to damage cell membranes but is able to inhibit macromolecular synthesis (Patrzykat et al., 2002). Antimicrobial substances have also been shown to cause membrane lysis, predominantly at high concentrations, and lysis without membranes - at low (Cudic \& Otvos et al., 2002). The importance of the concentration of a biologically active substance in the development of new treatments for priority diseases has been confirmed, and dose-effect curves have been proposed to accurately determine the optimal dose of the active component (García-Fuente et al., 2018). They assume that since most antibacterial substances have multiple target-cells, their mechanisms of action are directly proportional to concentration. The authors established the dependence of the rate (in time of death of the test cultures) of the antimicrobial activity of nisin and its combinations (with ciprofloxacin - for all strains, with vancomycin - for some strains) on its concentration (Dosler et al., 2011). According to our own preliminary data, the level of antimicrobial activity of metabolites of L. rhamnosus GG and $S$. boulardii depends on the initial concentration of the producers, which is used to obtain products of vital activity of probiotic cultures (Dosler et al., 2011). According to our preliminary data, the level of antimicrobial activity of metabolites of $L$. rhamnosus GG and S. boulardii depends on the initial concentration of the producer which is used to obtain products of vital activity of probiotic cultures.

The second area of research - is the combined use of lactobacteria and saccharomycetes with antibacterial drugs, presented in this publication, reproduced by the simultaneous effect of the derivatives and vital products of L. rhamnosus GG and S. boulardii and antibacterial preparations on Corynebacterium spp. tox ${ }^{+}$. The results of the synergistic effect confirm and supplement the literature on the potentiation of traditional antimicrobial agents with biologically active substances. Increased anti-staphylococcal activity is known with combined administration of the peptide ASU014 with oxacillin against Staphylococcus aureus. The expressed efficacy was caused by their joint use in comparison with individual drugs against the polyresistant pathogen (Lainson et al.,
2017). The ability of PMBN nanopeptide to synergise with antimicrobials in the presence of efflux inhibitors (efflux pump inhibitors) has been established. A study was performed on planktonic and biofilm formation cells of Pseudomonas aeruginosa strains with overexpression. Increased antimicrobial activity of azithromycin against this pathogen has been demonstrated (Ferrer-Espada et al., 2019). Membraneactive macromolecules, as well as metabolites, produced by their own methods, not only destroy planktonic and biofuel types of bacteria, but also potentiate antibiotics (erythromycin and rifampicin). The combination of macromolecules and antibiotics has been shown to significantly reduce bacterial loading in mouse burn models and infection of surgical wounds caused by Acinetobacter baumannii, carbapenemase and Klebsiella pneumoniae (Uppu et al., 2017). The use of molecule potentiators for the treatment of infections of bacterial origin by potentiation of antibiotics is known. They destroy the negatively charged outer membrane of resistant gram-negative microorganisms by allowing other impermeable molecules, including antibacterial drugs, to penetrate, providing susceptibility to pathogens with multiple drug resistance to a broader spectrum of antibiotics (Zabawa et al., 2016). The prospect of the use of microbial substances to enhance antibiotic activity is confirmed by scientists. Synergistic effect of polymyxin B and gramicidin S formed by Aneurinibacillus migulanus (Bacillus brevis), was established against 17 strains of Pseudomonas aeruginosa with multiple drug resistance and their biofilms. Combined application has opened new molecular targets in the membrane and impedes ligand binding on the hydrophobic surface of enzymes (Mogi \& Kita, 2009). In combination treatment with lipopeptide bacillomycin D and antifungal amphotericin B antimicrobial and wound healing effect against Candida albicans was established (Tabbene et al., 2016). The data obtained by the authors are consistent with our own results regarding the potentiation of antimicrobial activity of antibiotics against pathogenic microorganisms by the investigated substances.

The presented synergistic effects of filtrates of lactobacteria and saccharomycetes and antimicrobials are compared with the data of Sharma et al. on the potentiation of antibiotics by strains of Lactobacillus rhamnosus, L. acidophilus, Saccharomyces boulardii and Streptococcus faecalis (Sharma et al., 2014; Sharma \& Chauhan, 2014; Sharma \& Chauhan, 2015). Increased antimicrobial activity of aztreonam, amikacin, meropenem, ciprofloxacin by these probiotic cultures against reference and circulating $P$. aeruginosa strains was observed in $71.9 \%$ of the test samples (Sharma \& Chauhan, 2014). Synergistic effect is established when used together with aztreonam, amikacin, meropenem, ciprofloxacin (from 0 to $25 \mathrm{~mm}$ ). In subsequent work, the scientists have shown the increase in the effectiveness of amoxicillin/clavulanate when combined with probiotics $S$. boulardii and L. rhamnosus by $10 \mathrm{~mm}$ against $E$. coli. Increase of growth inhibition zones was established in $S$. aureus in $84.4 \%$ of samples, no change - in $12.5 \%$ and decrease - in 3.1\% samples of antibiotics (amoxicillin/clavulanate, azithromycin, ciprofloxacin) in combination with L. rhamnosus, S. boulardii, S. faecalis and L. acidophilus (Sharma et al., 2014; Sharma \& Chauhan, 2015). Comparing the experimental data presented in these articles with our own studies, it should be noted that it is necessary to use the same method to determine the effect of the tested substances on the antimicrobial activity of antibacterial preparations against pathogenic microorganisms - modified Kirby-Bauer disc diffusion method. The authors obtained the individual sensitivity of the test-cultures to the joint use of antibiotics with probiotic strains, which coincides with the presented results regarding the synergistic effect of filtrates of lactobacteria and saccharomycetes with antimicrobial preparations. The individual sensitivity of test-strains to biologically active substances of probiotic origin is also confirmed by our own previous data. Polyresistant gramnegative microorganisms (Pseudomonas aeruginosa, Klebsiella pneumonia, Lelliottia amnigena (Enterobacter amnigenus) showed the highest sensitivity to the metabolites of L. rhamnosus GG, and the pathogenic representatives of Corynebacterium tox ${ }^{+}-$to the combination of metabolites of L. rhamnosus GG and S. boulardii (Isayenko et al., 2019). In subsequent work, a team of scientists proved the enhancement of the anti-pseudomonad activity of colistin, polymyxin B by nisin a polypeptide antibiotic, formed by Lactococcus lactis (Field et al., 2016). 
A successful combination of nisin has been established with vancomycin and ciprofloxacin. Thus, synergistic interaction was observed with the use of nisin - ciprofloxacin in three of the five isolates, both resistant (MRSA) and susceptible (MSSA) to methicillin Staphylococcus aureus. The efficacy of the nisin-vancomycin combination was noted in two of the five strains of staphylococci (Dosler \& Gerceker, 2011). These data also coincide with our own findings regarding the individual susceptibility of pathogens to the combined use of biologically active substances with antimicrobial preparations.

The maximum increase in antimicrobial activity of the test combinations, according to the presented work, was observed with the combination of azithromycin, erythromycin, cefotaxime, ceftriaxone, ampicillin with metabolites of lactobacteria. The results of this work on the different degree of manifestation of synergistic effect depending on the activity of combinations, while simultaneously influencing them, are in good agreement with the data of studies of the following authors (Tong et al., 2014; Thomsen et al., 2016; Bolosov et al., 2017; Isayenko et al., 2019). Potentiation was noted of penicillin, ampicillin, gentamicin, kanamycin, roxithromycin, streptomycin, vancomycin, chloramphenicol, cefuroxime, cefazolin, ceftriaxone, cefepime, ciprofloxacin, imipenem and linezolid by nisin relative to three strains of Enterococcus faecalis. Separate use of roxithromycin, streptomycin, cefuroxime, cefazolin, ceftriaxone, cefepime was accompanied by low antimicrobial activity against $E$. faecalis cultures tested, and when combined with $200 \mathrm{U} / \mathrm{mL}$ nisin, there was a significant decrease in minimum inhibitory concentration and minimum bactericidal concentration of antibiotics. The best synergistic effect on the three $E$. faecalis strains was the combination of nisin and penicillin or chloramphenicol. Using a transmission electron microscope, it was found that $E$. faecalis was seriously impaired by any antibiotic in combination with nisin (Tong et al., 2014).

The presented results of the combined use of products of probiotics with antimicrobial preparations also coincide with the data of our previous studies on the antimicrobial properties of biologically active substances Lactobacillus rhamnosus GG and Saccharomyces boulardii. The expressed efficiency of structural components and metabolites of probiotic strains of lactobacteria and saccharomycetes in relation to pathogenic microorganisms, the degree of influence of which was dependent on the activity of the studied filtrate, was established (Isayenko et al., 2019). It is established that all substances of $L$. rhamnosus GG and $S$. boulardii, obtained by the author's method, have high antimicrobial activity against strains of Corynebacterium spp. tox ${ }^{+}$, and due to the co-culturing of L. rhamnosus GG and $S$. boulardii in the ultrasonic disintegrates of lactobacteria, the antimicrobial properties of the resulting vital products against pathogenic corynebacteria are increased. The combination of metabolites of lactobacteria and saccharomycetes also had the most pronounced anti-biofilm properties in relation to the biofilm formation of pathogens. And on the previously formed biofilms were more effective in metabolites of lactobacteria (Isayenko et al., 2019). The varying degree of synergistic effect of biologically active substances with antimicrobial drugs is also confirmed by the data of Bolosov et al. (2017). A study of the combined effect of arenicin-1 with a number of antibacterial drugs revealed several drug combinations with different levels of synergistic effect. A team of other researchers compared a number of peptide substances with pronounced antimicrobial activity proven in vitro tests and with their effectiveness in vivo studies. Most biologically active substances have not confirmed the antistaphylococcal properties in in vivo tests on Drosophila melanogaster. Only two lantibiotics, nisin and NAI-107, were bactericidal for both Staphylococcus aureus and the resistant strain MRSA USA300 and were not inferior to vancomycin efficacy (Thomsen et al., 2016). The intrinsic results are consistent with the data of this work regarding the high antimicrobial properties of biologically active substances isolated from Lactobacillus spp. The results of the study made it possible to determine that the final result is influenced by both the individual sensitivity of the test-strains of the test microorganisms and the level of antimicrobial activity of the biologically active substance used.

The consistent and simultaneous use of the structural components and metabolites of $L$. rhamnosus GG and $S$. boulardii to enhance the susceptibility of toxigenic strains of microorganisms and potentiation of existing antimicrobials is of great scientific importance. Increasing bioavailability may allow lower concentrations of antibacterial preparations to be used. And at storage of a therapeutic dose of an antibiotic, it is possible to shorten the term of its use through faster complete elimination of the pathogen. It is presumed that that due to the increased susceptibility of pathogenic bacteria, the development of resistance of microorganisms is inhibited.

\section{Conclusions}

The increase presented here in sensitivity of toxigenic strains of microorganisms to antimicrobial drugs is due to the consistent influence of the structural components and metabolites of L. rhamnosus GG and $S$. boulardii, obtained by the author's method, and antibacterial preparations on Corynebacterium spp. tox ${ }^{+}$. The greatest increase in the sensitivity of pathogenic test-cultures of corynebacteria after the use of biologically active substances of lactobacteria and saccharomycetes was observed in relation to penicillins (by 1.4-19.4 mm), carbapenems (by $1.0-15.0 \mathrm{~mm}$ ), then to the representative of glycopeptide antibiotic (by $0.2-12.0 \mathrm{~mm})$. The smallest inhibition of the diameter of the growth retardation zone of toxigenic representatives of the genus Corynebacterium was observed for the preparations of aminoglycosides (by 2.0 $11.0 \mathrm{~mm}$ ), macrolides (by 0.2-9.6 mm) and quinolones (by 2.0$9.8 \mathrm{~mm}$ ). Evaluation of the effect of filtrates of lactobacteria and saccharomycetes on the sensitivity of pathogenic bacteria to different groups of antibacterial preparations showed that the change in the sensitivity of the microbial cells of the pathogen was observed to varying degrees. Synergistic activity of ultrasonic disintegrates and products of vital activity of probiotic strains of microorganisms with traditional antibacterial preparations against Corynebacterium spp. strains has been proved thanks to the simultaneous nature of their impact. The maximal increase of antimicrobial activity of macrolides (by $4.4-4.6 \mathrm{~mm}$ ) was observed under the influence of metabolites of lactobacteria and the combination of metabolites of lactobacteria and saccharomycetes. The highest potentiation of beta-lactams $(0.2-2.2 \mathrm{~mm})$ relative to corynebacteria was observed by the filtrates of metabolites of lactobacteria. Different degrees of manifestation of joint action of the test substances with antibiotics were determined, which depended on the selected combinations, the method of influence on the microorganism, the activity of the filtrates of L. rhamnosus GG and S. boulardii, the individual sensitivity of the testcultures and the initial concentration of the producers which were used to obtain the metabolites of lactobacteria and saccharomycetes. The most active filtrates among all the samples for enhancing the antimicrobial activity of antibacterial preparations and increasing the sensitivity of corynebacteria to their combined use are the metabolites of lactobacteria, obtained by growing the producer with a high initial concentration (with optical density, corresponding to 10.0 units on the McFarland scale). Biologically active substances $L$. rhamnosus GG and S. boulardii, obtained without the use of traditional nutrient media, by increasing the bioavailability of pathogenic pathogens can reduce the required concentration of antibiotics, prolonging their use, and suspend the likelihood of the pathogens developing resistance to microorganisms, making them promising candidates for the development of nextgeneration "accompaniment-preparations" with the possibility of additional therapy for infectious diseases of different etiologies.

\section{References}

Atlas, R. (2010). Handbook of microbiological media. Boca Raton, London, New York.

Berditsch, M., Jäger, T., Strempel, N., Schwartz, T., Overhage, J., \& Ulrich, A. (2015). Synergistic effect of membrane-active peptides polymyxin B and gramicidin S on multidrug-resistant strains and biofilms of Pseudomonas aeruginosa. Antimicrobial Agents and Chemotherapy, 59(9), 5288-5296.

Bolosov, I., Kalashnikov, A., Panteleev, P., \& Ovchinnikova, T. (2017). Analysis of synergistic effects of antimicrobial peptide arenicin-1 and conventional antibiotics. Bulletin of Experimental Biology and Medicine, 162(6), 765-768.

Cassone, M., \& Otvos, L. (2010). Synergy among antibacterial peptides and between peptides and small-molecule antibiotics. Expert Review of Antiinfective Therapy, 8(6), 703-716. 
Chaudhary, A. (2016). A review of global initiatives to fight antibiotic resistance and recent antibiotics' discovery. Acta Pharmaceutica Sinica B, 6, 552-556.

Corbett, D., Wise, A., Langley, T., Skinner, K., Trimby, E., Birchall, S., Dorali, A., Sandiford, S., Williams, J., Warn, P., Vaara, M., \& Lister, T. (2017). Potentiation of antibiotic activity by a novel cationic peptide: Potency and spectrum of activity of SPR741. Antimicrobial Agents and Chemotherapy, 61, e00200-17.

Cudic, M., \& Otvos, L. (2002). Intracellular targets of antibacterial peptides. Current Drug Targets, 3(2), 101-106.

Dillon, N., Holland, M., Tsunemoto, H., Hancock, B., Cornax, I., Pogliano, J., Sakoulas, G., \& Nizet, V. (2019). Surprising synergy of dual translation inhibition vs. Acinetobacter baumannii and other multidrug-resistant bacterial pathogens. EBioMedicine, 46, 193-201.

Dosler, S., \& Gerceker, A. A. (2011). In vitro activities of nisin alone or in combination with vancomycin and ciprofloxacin against methicillin-resistant and methicillin-susceptible Staphylococcus aureus strains. Chemotherapy, 57(6), 511-516.

Ferrer-Espada, R., Shahrour, H., Pitts, B., Stewart, P. S., Sánchez-Gómez, S., \& Martínez-de-Tejada, G. (2019). A permeability-increasing drug synergizes with bacterial efflux pump inhibitors and restores susceptibility to antibiotics in multi-drug resistant Pseudomonas aeruginosa strains. Scientific Reports, 9(1), 3452.

Field, D., Seisling, N., Cotter, P. D., Ross, R. P., \& Hill, C. (2016). Synergistic nisin-polymyxin combinations for the control of Pseudomonas biofilm formation. Frontiers in Microbiology, 7, 1713.

Florin, T., Maracci, C., Graf, M., Karki, P., Klepacki, D., Beminghausen, O., Beckmann, R., Vázquez-Laslop, N., Wilson, D., Rodnina, M., \& Mankin, A. (2017) An antimicrobial peptide that inhibits translation by trapping release factors on the ribosome. Nature Structural and Molecular Biology, 24(9), 752-757.

García-Fuente, A., Vázquez, F., Viéitez, J. M., García Alonso, F. J., Martín, J. I., \& Ferrer, J. (2018). CISNE: An accurate description of dose-effect and synergism in combination therapies. Scientific Reports, 8(1), 4964.

Il'yashenko, M., Tarasova, G., \& Guseva, A. (2012). Endogennyye antimikrobnyye peptidy i ikh kliniko-patogeneticheskaya znachimost' pri vospalitel'nykh zabolevaniyakh kishechnika [Endogenic antimicrobial peptides and their clinical-pathogenetic significance in inflammatory diseases of the intestine]. Modern Problems of Science and Education, 2 (in Russian).

Isayenko, O., Knysh, O., Kotsar, O., Ryzhkova, T., \& Dyukareva, G. (2019). Evaluation of anti-microbial activity of filtrates of Lactobacillus rhamnosus and Saccharomyces boulardii against antibiotic-resistant gram-negative bacteria. Regulatory Mechanisms in Biosystems, 10(2), 245-250.

Isayenko, O., Knysh, O., Babych, Y., Ryzhkova, T., \& Dyukareva, G. (2019). Effect of disintegrates and metabolites of Lactobacillus rhamnosus and Saccharomyces boulardii on biofilms of antibiotic resistant conditionally pathogenic and pathogenic bacteria. Regulatory Mechanisms in Biosystems, 10(1), 3-8.

Isajenko, O., Knysh, O., Fal'ko, O., Prokopyuk, V., \& Prokopyuk, O. (2019). Tsytotoksychnosti strukturno-metabolitnykh kompleksiv Lactobacillus rhamnosus GG ta Saccharomyces boulardii [Cytotoxicity structural-metabolitic complexes of Lactobacillus rhamnosus GG and Saccharomyces boulardii]. Fiziolohichnyi Zhurnal, 65(5), 35-41 (in Ukrainian).

Ismeeal, M. C., Ibrahim, K. M., \& Al-Malikey, M. K. (2013). The effect of surlactin produced by Lactobacillus acidophilus on eye infectious bacteria in rabbits. Baghdad Science Journal, 10(1), 133-143.

Jing, H., Charles, G., William, C., \& Wimley. (2015). A lack of synergy between membrane-permeabilizing cationic antimicrobial peptides and conventional antibiotics. Biochimica et Biophysica Acta, 1848(1), 8-15.

Lainson, J. C., Daly, S. M., Triplett, K., Johnston, S. A., Hall, P. R., \& Diehnelt, C. W. (2017). Synthetic antibacterial peptide exhibits synergy with oxacillin against MRSA. ACS Medicinal Chemistry Letters, 8(8), 853-857.

Lewies, A., Du Plessis, L., \& Wentzel, J. (2018). Antimicrobial peptides: The achilles' heel of antibiotic resistance? Probiotics and Antimicrobial Proteins, $11,370-381$.

Li, X.-Z., Plésiat, P., \& Nikaido, H. (2015). The challenge of efflux-mediated antibiotic resistance in Gram-negative bacteria. Clinical Microbiology Reviews, 28 (2), 337-418

Lin, L., Nonejuie, P., Munguia, J., Hollands, A., Olson, J., Dam, Q., Kumaraswamy, M., Rivera, H., Corriden, R., Rohde, M., Hensler, M., Burkart, M., Pogliano, J., Sakoulas, G., \& Nizet, V. (2015). Azithromycin synergizes with cationic antimicrobial peptides to exert bactericidal and therapeutic activity against highly multidrug-resistant gram-negative bacterial pathogens. EBioMedicine, 2(7), 690-698

Mardirossian, M., Grzela, R., Giglione, C., Meinnel, T., Gennaro, R., Mergaert, P. \& Scocchi, M. (2014). The host antimicrobial peptide bac71-35binds to bacterial ribosomal proteins and inhibits protein synthesis. Cell Chemical Biology, 21(12), 1639-1647.

Metelev, M., Osterman, I. A., Ghilarov, D., Khabibullina, N. F., Yakimov, A., Shabalin, K., Utkina, I., Travin, D. Y., Komarova, E. S., Serebryakova, M., Artamonova, T., Khodorkovskii, M., Konevega, A. L., Sergiev, P. V., Severinov,
K., \& Polikanov, Y. S. (2017). Klebsazolicin inhibits 70S ribosome by obstructing the peptide exit tunnel. Nature Chemical Biology, 13, 1129-1136.

Mogi, T., \& Kita, K. (2009). Gramicidin S and polymyxins: The revival of cationic cyclic peptide antibiotics. Cellular and Molecular Life Sciences, 66(23), 3821-3826.

Morici, P., Florio, W., Rizzato, C., Ghelardi, E., Tavanti, A., Rossolini, G. M., \& Lupetti, A. (2017). Synergistic activity of synthetic N-terminal peptide of human lactoferrin in combination with various antibiotics against carbapenem-resistant Klebsiella pneumoniae strains. European Journal of Clinical Microbiology and Infectious Diseases, 36(10), 1739-1748.

Mulani, M., Kamble, E., Kumkar, S. N., Tawre, M. S., \& Pardesi, K. R. (2019) Emerging strategies to combat ESKAPE pathogens in the era of antimicrobial resistance: A review. Frontiers in Microbiology, 10, 539

Nagarajan, D., Roy, N., Kulkami, O., Nanaikar, N., Datey, A., Ravichandran, S. Thakur, C. S., Aprameya, I. V., Sarma, S. P., Chakravortty, D., \& Chandra, N. (2019). $\Omega 76$ : A designed antimicrobial peptide to combat carbapenem- and tigecycline-resistant Acinetobacter baumannii. Science Advances, 5(7), 1946.

Patrzykat, A., Friedrich, C. L., Zhang, L., Mendoza, V., \& Hancock, R. E. (2002). Sublethal concentrations of pleurocidin-derived antimicrobial peptides inhibit macromolecular synthesis in Escherichia coli. Antimicrobial Agents and Chemotherapy, 46(3), 605-614.

Pfalzgraff, A., Brandenburg, K., \& Weindl, G. (2018). Antimicrobial peptides and their therapeutic potential for bacterial skin infections and wounds. Frontiers in Pharmacology, 9, 281.

Pizzolato-Cezar, L., Okuda-Shinagawa, N., \& Machini, M. (2019). Combinatory therapy antimicrobial peptide-antibiotic to minimize the ongoing rise of resistance. Frontiers in Microbiology, 10, 1703

Pletzer, D., Mansour, S. C., \& Hancock, R. E. W. (2018). Synergy between conventional antibiotics and anti-biofilm peptides in a murine, sub-cutaneous abscess model caused by recalcitrant ESKAPE pathogens. PLoS Pathogens, 14(6), e1007084

Pollini, S., Brunetti, J., Sennati, S., Rossolini, G., Bracci, L., \& Pini, A. (2017). Synergistic activity profile of an antimicrobial peptide against multidrug-resistant and extensively drug-resistant strains of gram-negative bacterial pathogens. Journal of Peptide Science, 23, 329-333.

Reffuveille, F., de la Fuente-Núñez, C., Mansour, S., \& Hancock, R. E. W. (2014). A broad-spectrum antibiofilm peptide enhances antibiotic action against bacterial biofilms. Antimicrobial Agents and Chemotherapy, 58, 5363-5371.

Ribeiro, S. M., de la Fuente-Núñez, C., Baquir, B., Faria-Junior, C., Franco, O. L., \& Hancock, R. E. (2015). Antibiofilm peptides increase the susceptibility of carbapenemase-producing Klebsiella pneumoniae clinical isolates to $\beta$-lactam antibiotics. Antimicrobial Agents and Chemotherapy, 59(7), 3906-3912

Richardson, L. (2017). Understanding and overcoming antibiotic resistance. PLoS Biology, 15(8), e2003775.

Satpute, S., Kulkarni, G., Banpurkar, A., \& Banat, I. M. (2016). Biosurfactant's from Lactobacteria species: Properties, challenges and potential biomedical applications: Biosurfactant's from Lactobacteria species. Journal of Basic Microbiology, 56(11), 1140-1158.

Sharma, J., \& Chauhan, D. S. (2014). Inhibition of Pseudomonas aeruginosa by antibiotics and probiotics combinations - In vitro study. European Journal of Experimental Biology, 4(6), 10-14.

Sharma, J., \& Chauhan, D. S. (2015). In vitro study on the role of probiotic strains in potentiation of antimicrobial activity against Staphylococcus aureus. International Journal of Pharmacy and Life Sciences, 6(1), 4161-4165.

Sharma, J., Chauhan, D. S., \& Goyal, A. (2014). Enhancement of antimicrobial activity of antibiotics by probiotics against Escherichia coli - An in vitro study. Advances in Applied Science Research, 5(6), 14-18.

Tabbene, O., Azaiez, S., Di Grazia, A., Karkouch, I., Ben Slimene, I., Elkahoui, S., Alfeddy, M. N., Casciaro, B., Luca, V., Limam, F., \& Mangoni, M. L. (2016). Bacillomycin D and its combination with amphotericin B: Promising antifungal compounds with powerful antibiofilm activity and wound-healing potency. Journal of Applied Microbiology, 120(2), 289-300.

Tegos, G. P., Haynes, M., Strouse, J., Khan, M. T., Bologa, C. G., Oprea, T. I., \& Sklar, L. A. (2011). Microbial efflux pump inhibition: Tactics and strategies. Current Pharmaceutical Design, 17(13), 1291-1302.

Thomsen, T., Mojsoska, B., Cruz, J., Donadio, S., Jenssen, H., Løbner-Olesen, A., \& Rewitz, K. (2016). The lantibiotic NAI-107 efficiently rescues Drosophila melanogaster from infection with methicillin-resistant Staphylococcus aureus USA300. Antimicrobial Agents and Chemotherapy, 60(9), 5427-5436.

Tong, Z., Zhang, Y., Ling, J., Ma, J., Huang, L., \& Zhang, L. (2014). An in vitro study on the effects of nisin on the antibacterial activities of 18 antibiotics against Enterococcus faecalis. PLoS One, 9(2), e89209.

Uppu, D., Konai, M. M., Sarkar, P., Samaddar, S., Fensterseifer, I., Farias-Junior, C., Krishnamoorthy, P., Shome, B. R., Franco, O. L., \& Haldar, J. (2017). Membrane-active macromolecules kill antibiotic-tolerant bacteria and potentiate antibiotics towards Gram-negative bacteria. PLoS One, 12(8), e0183263.

Van Bambeke, F., Pagès, J., \& Lee, V. J. (2006). Inhibitors of bacterial efflux pumps as adjuvants in antibiotic treatments and diagnostic tools for detection 
of resistance by efflux. Recent Patents on Anti-Infective Drug Discovery, $1(2), 157-175$.

Volyanskiy, Y., Biryukova, S., Shapovalova, O., Stegniy, B., Manina, Z., \& Gorbatenko, S. (2014). Korinebakterii. Rol' v patologii cheloveka i zhivotnykh [Corynebacterium. Role in human and animal pathology]. FOP Brovin, Kharkov (in Ukrainian).

Xu, X., Xu, L., Yuan, G., Wang, Y., Qu, Y., \& Zhou, M. (2018). Synergistic combination of two antimicrobial agents closing each other's mutant selection windows to prevent antimicrobial resistance. Scientific Reports, 8(1), 7237.

Yasir, M., Dutta, D., \& Willcox, M. D. P. (2019). Comparative mode of action of the antimicrobial peptide melimine and its derivative Mel4 against Pseudomonas aeruginosa. Scientific Reports, 9(1), 7063.

Wang, S., Wang, Q., Zeng, X., Ye, Q., Huang, S., Yu, H., Yang, T., \& Qiao, S. (2017). Use of the antimicrobial peptide sublancin with combined antibacterial and immunomodulatory activities to protect against methicillin-resistant Staphylococcus aureus infection in mice. Journal of Agricultural and Food Chemistry, 65(39), 8595-8605.

Wenzel, M., Chiriac, A., Otto, A., Zweytick, D., May, C., Schumacher, C., Gust, R., Albada, H., Penkova, M., Krämer, U., Erdmann, R., Metzler-Nolte, N., Straus, S. K., Bremer, E., Becher, D., Brötz-Oesterhelt, H., Sahl, H., \& Ban- dow, J. (2014). Small cationic antimicrobial peptides delocalize peripheral membrane proteins. Proceedings of the National Academy of Sciences, 111(14), 409-418.

World Health Organization (2017). Global priority list of antibiotic-resistant bacteria to guide research, discovery, and development of new antibiotics. WHO.

World Health Organization (2017). Antibacterial agents in clinical development: An analysis of the antibacterial clinical development pipeline, including $M y$ cobacterium tuberculosis. WHO.

World Health Organization (2018). 2018: Ten threats to human health this year. WHO.

World Health Organization (2018). Weekly epidemiological bulletin, 23(93), 329-344.

Wu, X., Li, Z., Li, X., Tian, Y., Fan, Y., \& Yu, C. (2017). Synergistic effects of antimicrobial peptide DP7 combined with antibiotics against multidrugresistant bacteria. Drug Design, Development and Therapy, 11, 939-946.

Zabawa, T. P., Pucci, M. J., Parr, T. R., \& Lister, T. (2016). Treatment of gramnegative bacterial infections by potentiation of antibiotics. Current Opinion in Microbiology, 33, 7-12. 